\title{
Plastic Optical Fibre Sensing of Fuel Leakage in Soil
}

\author{
Masayuki Morisawa and Shinzo Muto \\ Department of Information and Communication System, Graduate School of Medicine and Engineering, University of Yamanashi, \\ 4-3-11 Takeda, Kofu 400-8511, Japan \\ Correspondence should be addressed to Masayuki Morisawa, morisawa@yamanashi.ac.jp
}

Received 1 December 2011; Accepted 13 February 2012

Academic Editor: Kevin Chen

Copyright ( $) 2012$ M. Morisawa and S. Muto. This is an open access article distributed under the Creative Commons Attribution License, which permits unrestricted use, distribution, and reproduction in any medium, provided the original work is properly cited.

\begin{abstract}
A basic operation of the very simple optical sensing system of fuel leakage in uniform sandy and clayey soils, which is consisting of a plastic optical fibre (POF) transmission line, the POF-type sensor heads, and a single LED photodiode pair, has been studied theoretically and experimentally. Its sensing principle is based on the POF structure change in the sensor head caused by fuels such as petrol. A scale-downed model prepared in the experimental room showed a possibility of optical detection of fuel leakage points in uniform soil. As this system operates without receiving the influence of water containing in fuels and soils, its application to fuel leak monitor around a filling station and oil tank can be expected.
\end{abstract}

\section{Introduction}

At present, various fuels such as petrol, kerosene, and light oil are used in many fields to make our life comfortable. However, leakage of fuels produces many serious problems such as pollution in soil or underwater, explosion, and combustion in a filling station or oil tank. To prevent such serious problems, quick and safety detection of fuel leakage point is strongly required. Of course, it goes without saying that an optical sensing is the safest method $[1,2]$, and, hitherto, several optical sensing systems such as an optical time domain reflectometry (OTDR) and a multipoint sensor system have been studied $[3,4]$. However, considering the practical application around a filling station and oil tank, more simple and low-cost ones are desired. From this background, we made an attempt to develop a very simple and low-cost optical sensing system to detect leakage of fuel in soil. Therefore, we mainly used the POF because it has many advantages such as low cost, easiness in handling, and immunity to electromagnetic interference [5]. Its system consists of the POF transmission line, several POF-type sensor heads, and a single LED photodiode pair. According to the preliminary study, certain kinds of polymer such as polyisoprene (PIP) cause swelling by attachment of fuel molecules and then change its refractive index remarkably
[6]. Based on this phenomenon, the POF-type sensor head to detect petrol was fabricated and tested. Specially, a scaledowned model of the sensing system for detecting fuel leakage in uniform sandy and clayey soils has been studied theoretically and experimentally. This paper reports on its basic property towards development of the practical system.

\section{Application Model and Its Operation Principle}

The model considered here is shown schematically in Figure 1. As can be seen from this figure, the proposed optical sensing system consists of the POF transmission line, several POF-type sensor heads, and a single LED photodiode pair. In addition, its system assumes that the POF is buried underground but is not subjected to such a large pressure that would deform it considerably by breaking and crushing it to the extent that its original shape and state cannot be restored. This model seems to be suitable for the practical case around a filling station or oil tank. The sensing principle in the POF-type sensor head is based on the swell phenomenon in its cladding layer. When is exposed to vapour phase alkane or petrol, the refractive index of the PIP film with a thickness of about $5 \mu \mathrm{m}$ decreases from 1.52 to 1.48 as shown in Figure 2. The solid line in Figure 2 shows 


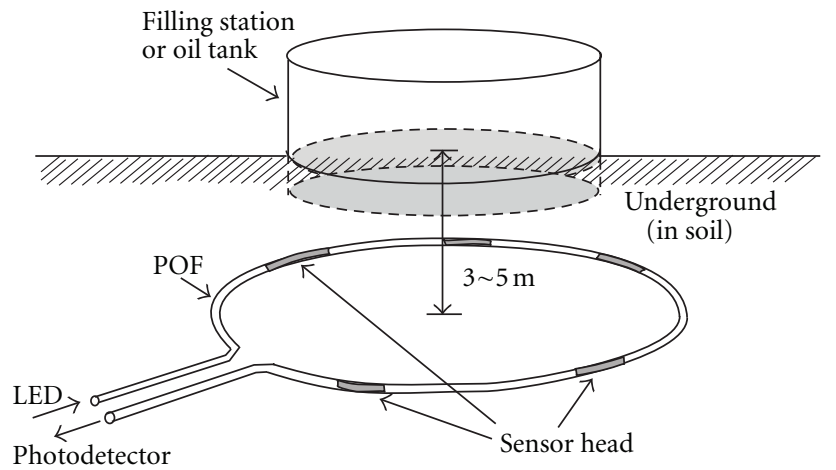

Figure 1: Application model of the POF-type sensing system.

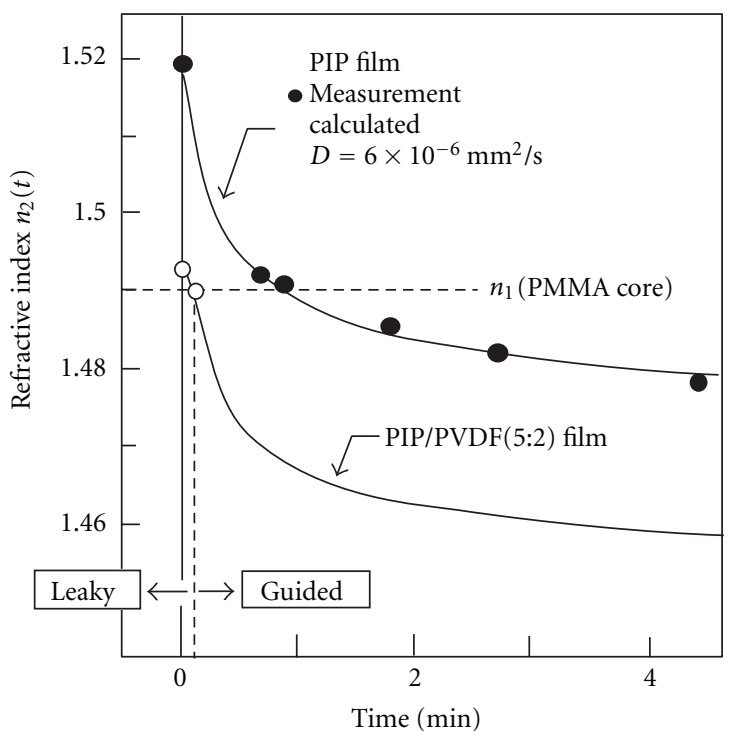

Figure 2: Refractive index changes in PIP and PIP/PVDF $(5: 2)$ films after exposing to hexane vapour.

the theoretical change in the refractive index $n(t)$, which is calculated using a following one-dimensional diffusion equation for hexane concentration $N(t)$ in the PIP film with a diffusion coefficient of $D=6 \times 10^{-6} \mathrm{~mm} / \mathrm{s}$ and a relation between $N(t)$ and $n(t)[7,8]$ :

$$
\frac{\partial N(x, t)}{\partial t}=D \frac{\partial^{2} N(x, t)}{\partial x^{2}} .
$$

Assuming the initial condition of $N(x, 0)=0$ and $N(0, t)=N_{0}$, the solution of above one dimensional diffusion equation is easily obtained as follows:

$$
N(x, t)=N_{0}\left(1-\frac{2}{\sqrt{\pi}} \int_{0}^{x /(2 \sqrt{D t})} e^{-\alpha^{2}} d \alpha\right) .
$$

And then, as the density in the swelling film decreases with increasing $N(x, t), n(x, t)$ can be expressed by the following equation:

$$
n(x, t)=\frac{n_{0} n_{\infty}\left(N_{0}\right)}{\left(n_{0}-n_{\infty}\left(N_{0}\right)\right) N(x, t) / N_{0}+n_{\infty}\left(N_{0}\right)},
$$

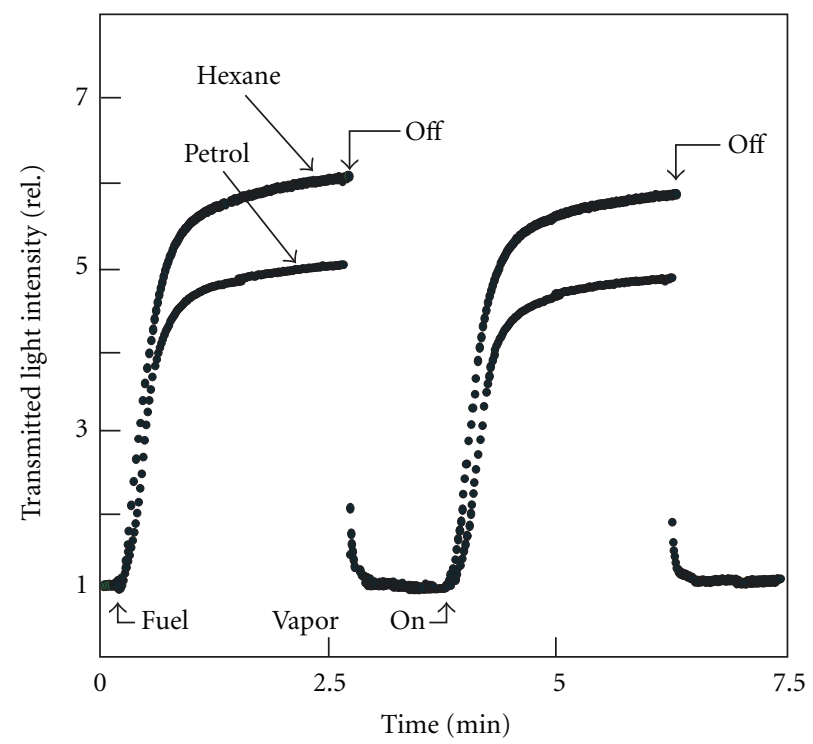

FIgure 3: Response of POF-type sensor head with the PIP/PVDF $(5: 2)$ cladding layer to hexane and petrol vapours.

where $n_{0}$ is the refractive index at $N(x, 0)$ and $n\left(N_{0}\right)$ is the final one at $N(x, \infty)=N_{0}$.

Considering the POF structure change in the sensor head consisting of the swell polymer clad and polymethylmethacrylate (PMMA) core with a refractive index of $n_{1}=1.490$, the initial refractive index $\left(n_{2}\right)$ in the cladding layer must be set at a slightly lager value than $n_{1}$ in the fibre core [8]. Therefore, the mixture of the PIP and polyvinylidenfluoride (PVDF) with a ratio of 5 to 2 was considered as a sensitive cladding layer. The bare PMMA core was obtained by removing the cladding layer from the commercialized POF without a jacket (Mitsubushi-Rayon, ESKA), by mean of organic solvent such as 1,4-dioxane. About 15 minutes immersion at $20^{\circ} \mathrm{C}$ and wiping off the dissolved cladding polymer using a soft tissue made us easily obtain the bare PMMA core with a smooth surface. The PIP/PVDF $(5: 2)$ cladding polymer was dissolved in dimethylsulfoxide (DMSO) and was dip-coated on the bare PMMA core with a diameter of about $1 \mathrm{~mm}$. Its thickness is about $5 \mu \mathrm{m}$. According to these conditions, the repeatability of the sensor 


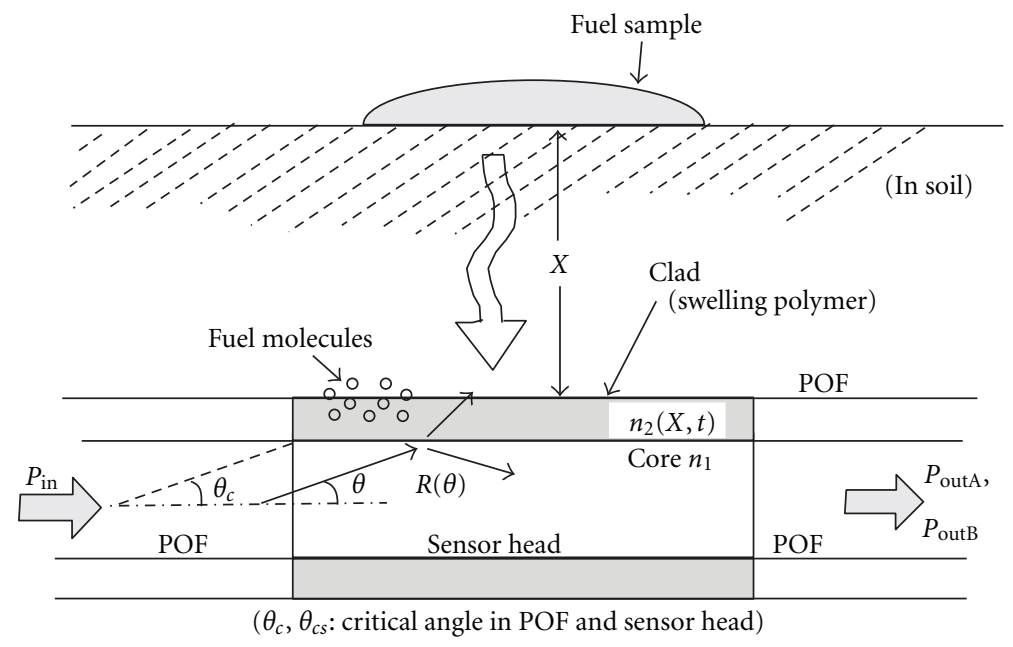

(A) Without fuel sample;

$n_{2}>n_{1}$ (Leaky POF)

$R(\theta) \ll 1\left(0<\theta<\theta_{c}\right)$
(B) With fuel sample;

$n_{2}<n_{1}$ (guided POF)

$R(\theta)=1\left(0<\theta<\theta_{c s}\right)$

$\ll 1\left(\theta_{c s}<\theta<\theta_{c}\right)$

FIGURe 4: POF sensing model to detect fuel leakage in uniform soil. $P_{\text {outA }}$ is the output light intensity, and $P_{\text {outB }}$ is that one with fuel sample.

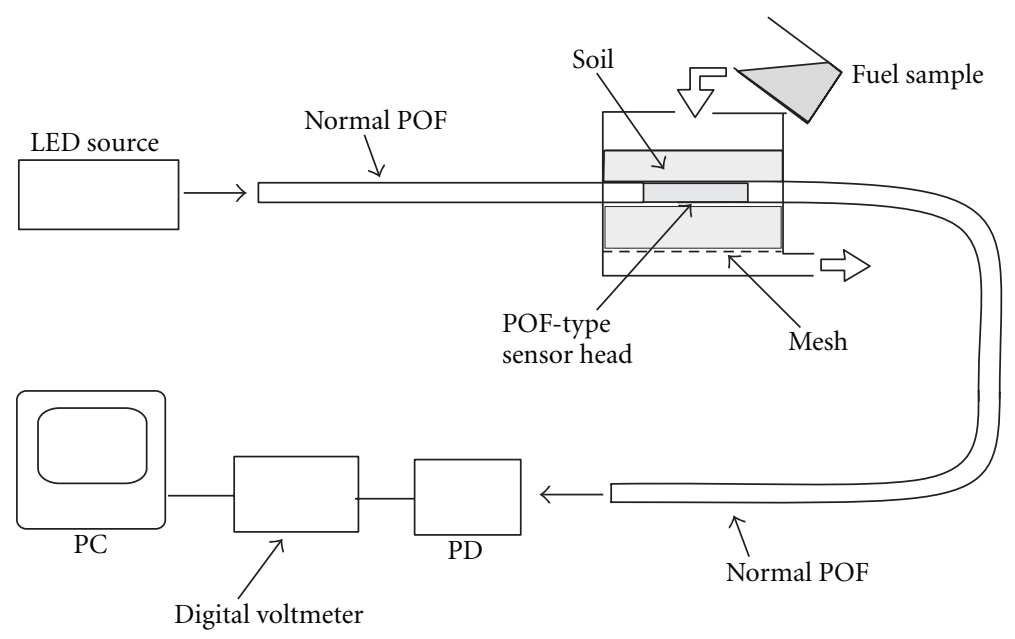

Figure 5: Experimental setup.

fabrication becomes very high. When was exposed to hexane vapour, its refractive index in cladding layer changed from 1.492 to 1.455 , as also shown in Figure 2. From this result, it is clear that the POF-type sensor head changes quickly its structure from a leaky type to a guided one.

First, the sensor head with a length of $50 \mathrm{~mm}$ was tested to confirm its operation even in the wavelength region with a large propagation loss. So a blue LED was used as a light source. Figure 3 shows the results for vapour phase hexane and petrol. As can be seen from this figure, a large change in transmitted light intensity based on the POF structure change from leaky type to guided one was observed experimentally. In addition, the proposed sensor structure gave high sensitivity and good reproducibility for both petrol and hexane. Referring to these results, the sensor head with a length of $10 \mathrm{~mm}$ was connected to the normal POFs at the input and output edges and was set at the position $x=55 \mathrm{~mm}$ in uniform sandy soil or clayey soil as shown in Figure 4. Here, the light reflection at the cladsoil boundary was neglected to simplify the analysis. In this figure, for the case of (a) without fuel sample, the output light intensity $P_{\text {outA }}$ is small because the power reflection coefficient $R(\theta)$ at the core-clad boundary becomes a very small value [8]. On the other hand, for the case of (b) with fuel sample, the output light intensity $P_{\text {outB }}$ increases remarkably because of its change to the guided structure. Then, the value of $P_{\text {outB }} / P_{\text {out }}$ gives the change in transmitted light intensity, that is, the sensitivity. Its property 


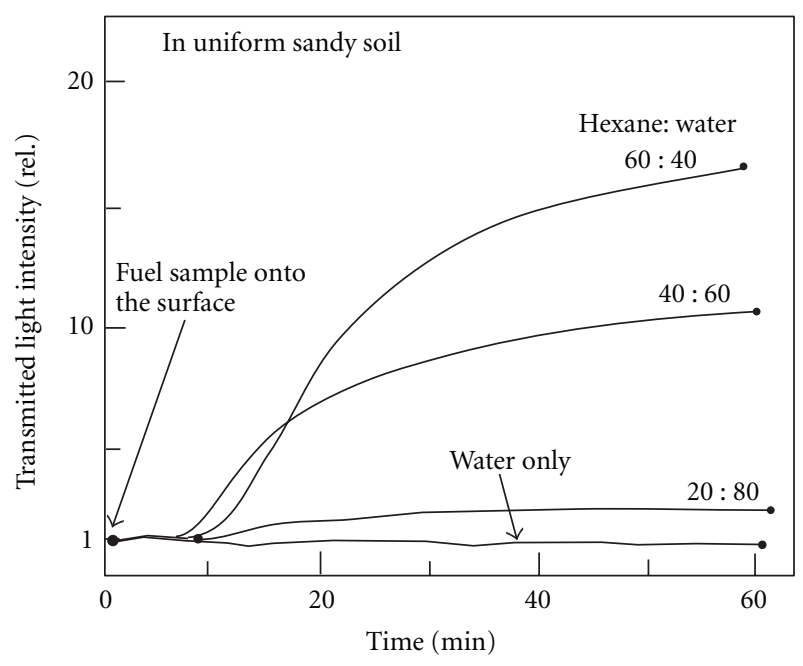

Figure 6: Sensor response observed in uniform sandy soil.

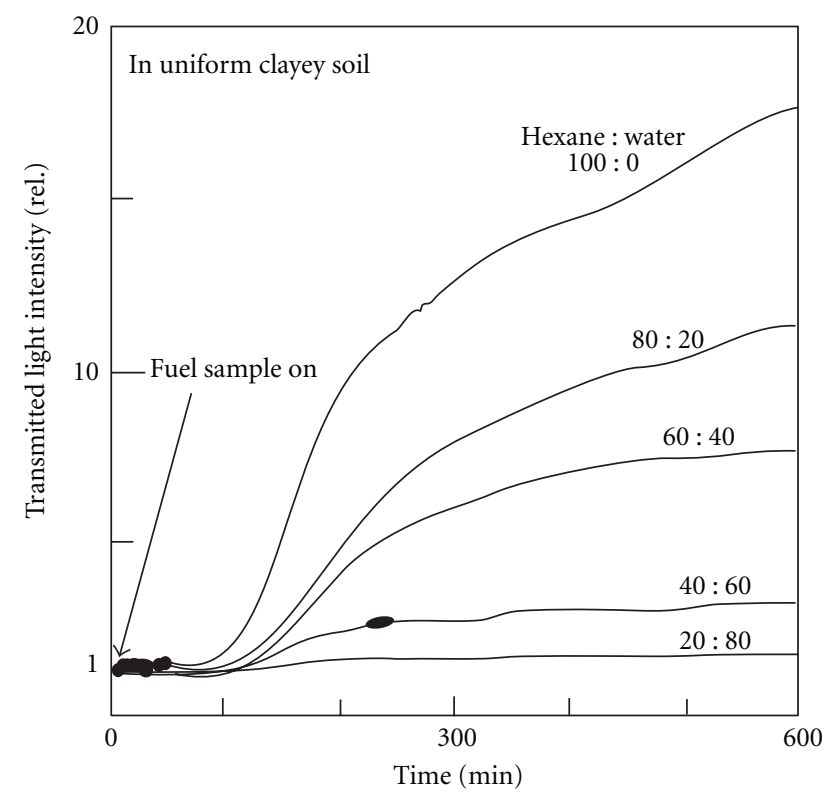

FIGURE 7: Sensor response observed in uniform clayey soil.

was measured using a photodetector, and its electrical signal was fed to a digital voltmeter connected to a computer for real-time data processing, as shown in Figure 5. In this experiment, to check an influence of water, the mixture of hexane and water was used as the fuel sample [7]. The obtained sensor responses are shown in Figures 6 and 7, respectively. From these figures, it is clear that the change in transmitted light intensity increases depending on the fuel concentration without receiving the influence of water and that diffusion of the fuel sample in clayey soil takes a much longer time. Furthermore, the diffusion coefficient of fuel sample in uniform soil was estimated from the start time of increase in the transmitted light intensity. For example, its value in uniform sandy soil used here was decided to be $0.7 \mathrm{~mm}^{2} / \mathrm{s}$. Using this value and a ray tracing method

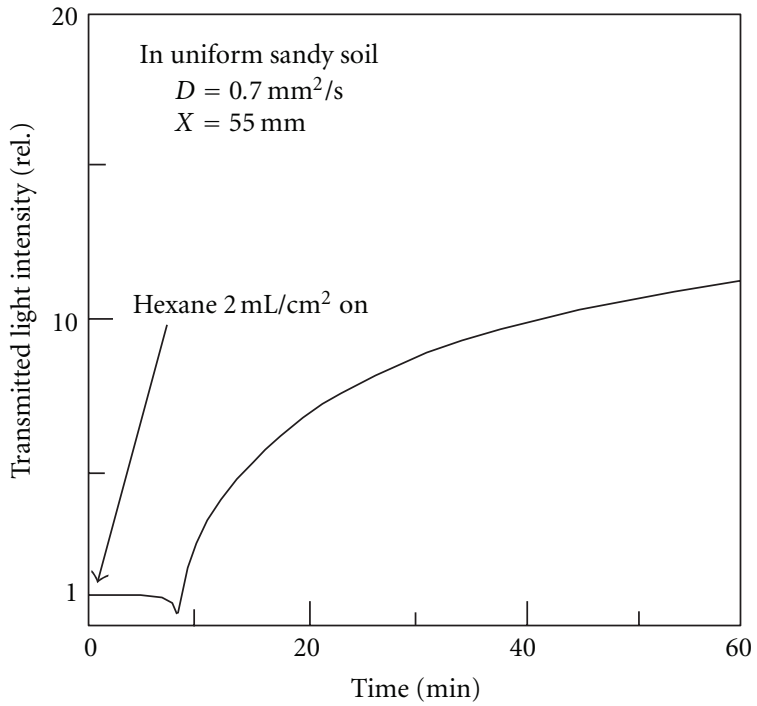

FIGURE 8: Calculated sensor response in uniform sandy soil.

[8], the change in transmitted light intensity through the sensor head can also be calculated theoretically. Its result is shown in Figure 8. As is clear from the figure, excepting the small dip at the critical point caused by the neglect of light reflection at the clad-soil boundary, the calculated sensor response coincided qualitatively with the experimental ones shown in Figure 6. In addition, as the similar operation was confirmed in the case with a long sensor head of $120 \mathrm{~mm}$, a series connection of several sensor heads with a total length below $120 \mathrm{~mm}$ seems to be possible.

\section{Detection Method of Fuel Leakage Point}

Considering a practical application of the above sensor, detection of the leakage point is needed. Therefore, the POF sensing system with multisensor heads was considered. Its system is shown schematically in Figure 9, in which, the upper configuration is type (a) with equal sensor head lengths of $10 \mathrm{~mm}$ and the lower one is type (b) with different sensor head lengths of $30 \mathrm{~mm}, 20 \mathrm{~mm}$, and $10 \mathrm{~mm}$, respectively. This model also means a scale-downed sensor model of the fuel leakage point around a filling station or oil tank shown in Figure 1. However, to shorten the experiment time, these sensor heads were buried in uniform light sandy soil with a diffusion coefficient of $D_{1}=80 \mathrm{~mm}^{2} / \mathrm{s}$ and at $120 \mathrm{~mm}$ in depth. The value of $D_{1}$ was estimated experimentally using the same method described in Section 2. Furthermore, considering the directional diffusion of petrol in uniform light sandy soil, these values of $D_{2}$ and $D_{3}$ were also estimated from the value of $D_{1}$ to be $30 \mathrm{~mm}^{2} / \mathrm{s}$ and $10 \mathrm{~mm}^{2} / \mathrm{s}$, respectively. Under these conditions, a certain amount of petrol was poured onto the surface points marked with the closed circles of A, B, and C, respectively. To simplify the analysis, we assumed that fuel leakages from each point do not occur at the same time. Then, we can consider the above system as a cascade connection of three sensor heads. The calculated transmitted light intensity changes for each 


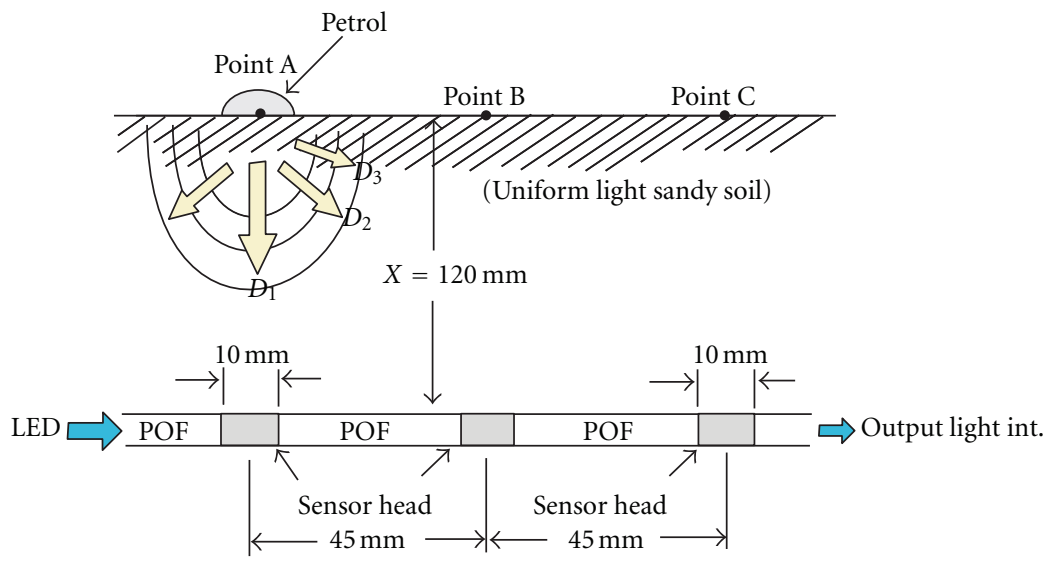

(a)

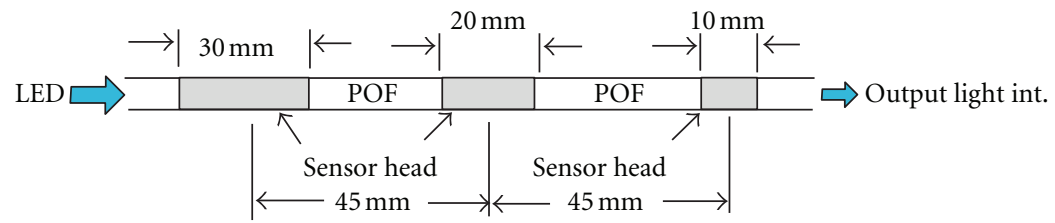

(b)

FIGURE 9: Three sensor heads system to detect petrol leakage point in uniform light sandy soil. (a) With equal sensor head lengths of $10 \mathrm{~mm}$ and (b) with sensor head lengths of $30 \mathrm{~mm}, 20 \mathrm{~mm}$, and $10 \mathrm{~mm}$.

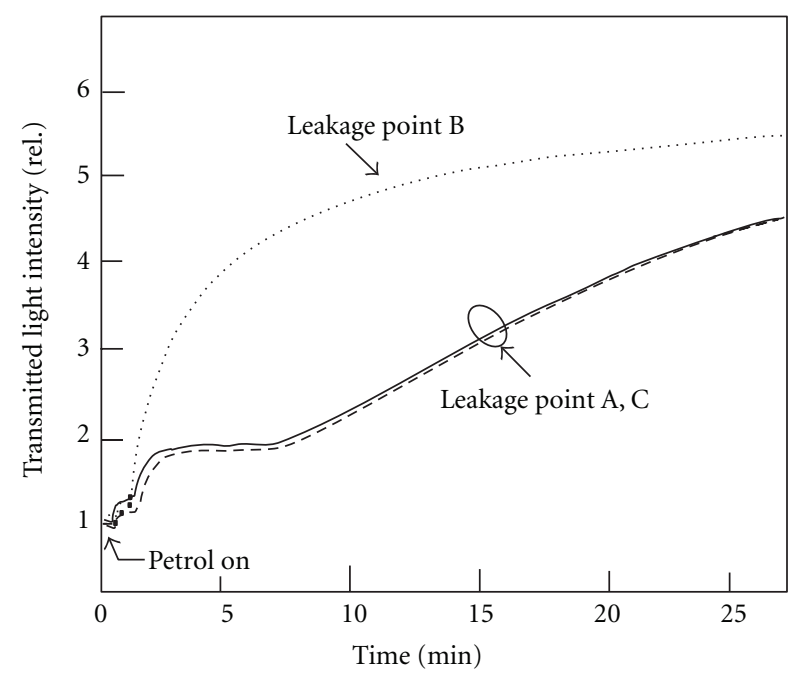

Figure 10: Calculated output light intensity change in type (a) for each leakage point.

leakage point A, B, and C are shown in Figures 10 and 11, respectively. From these figures, it is found that the change in light intensity for the leakage point $\mathrm{A}$ or $\mathrm{C}$ becomes small in the early stage but that one for the fuel leakage point $\mathrm{B}$ increases remarkably. The later property is due to that, a few minute after the petrol reaching to the second sensor head, the first and the third sensor heads operate as a guided POF at almost same time. In addition, the difference of the sensor head length between the first sensor head and third one made clear the change in the light intensity level in the early stage.

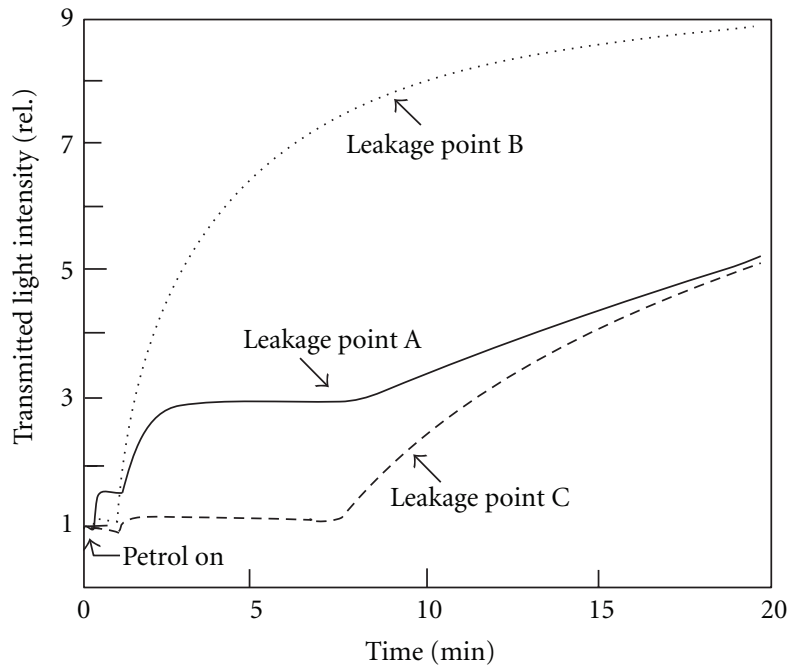

FIGURE 11: Calculated output light intensity change in type (b) for each leakage point.

Judging from these results, the system of type (b) showed a useful property to detect fuel leakage point.

To confirm the above characteristics experimentally, the same configuration of the three sensor heads and measurement setup were used. In this experiment, the transmitted light intensity changes in only early stage were measured because the continuous supply of petrol was difficult. The results obtained are shown in Figures 12 and 13, respectively. As is clear from these figures, the experimental properties in 


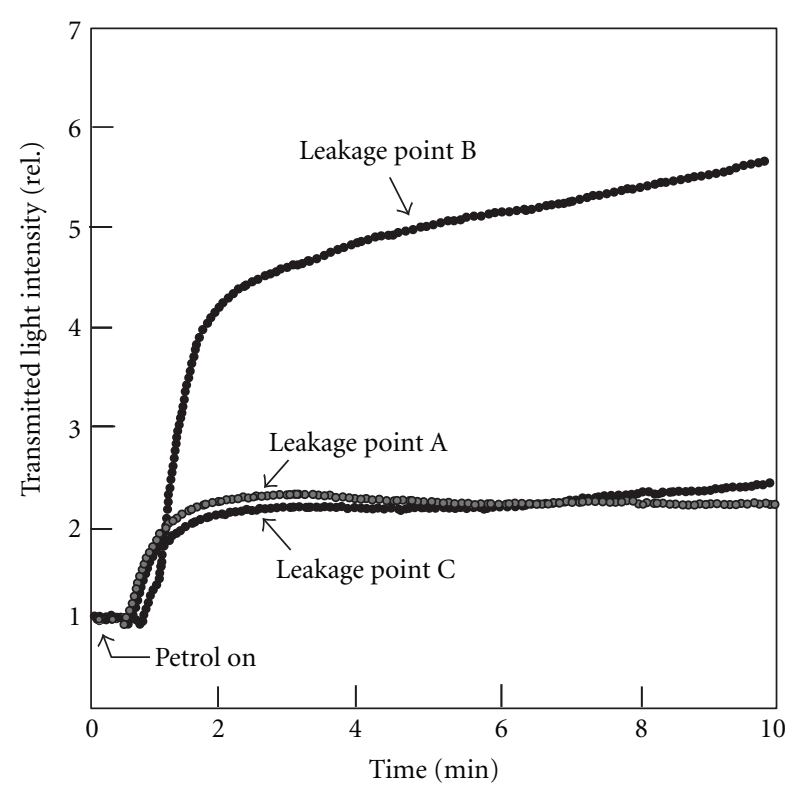

FIGURE 12: Observed light intensity change in early stage for type (a).

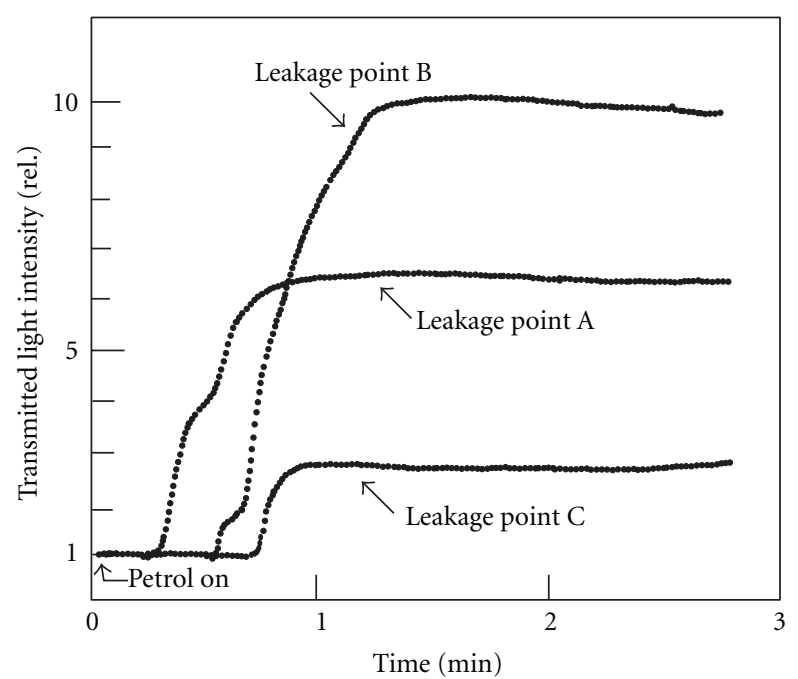

FIGURE 13: Observed light intensity change in early stage for type (b).

early stage of fuel leakage coincided qualitatively with the calculated ones. Thus, a very simple system consisting of the POF transmission line and the POF-type sensor head showed a possibility on optical detection of the fuel leakage point in uniform soil around a filling station and/or oil tank. In addition, this system will be used over whole visible region because it operated even in the blue wavelength. If the fuel leakages from some points occur simultaneously, a complicated change in the transmitted light intensity will be observed. Such a response also gives an important information showing the simultaneous fuel leakage from some points. Furthermore, when considering the practical uses of this sensor, it should be noted that this sensor system depends significantly on the diffusion coefficient of soil into gasoline. Therefore, for practical uses of this sensor, calibration should be performed by collecting a soil sample from the site where this system is set up and measuring the diffusion coefficient of the soil sample. However, when the diffusion coefficient is not uniform, the sensor response may become complicated. Therefore, we wish to treat them theoretically and experimentally in the near future.

\section{Conclusions}

The very simple POF-type optical sensing system of fuel leakage in uniform soils, which is based on the POF structure change in the sensor head produced by a swell phenomenon in the cladding layer, has been studied using a scale-downed model. Its theoretical and experimental results showed that a quick and safety detection of petrol leakage in uniform soil becomes possible without receiving an influence of water included in fuel and soil. Although this system is not suitable for the distributed leakage detection over a long distance, it seems to be used for a few point detection around a filling station and/or oil tank. Referring to these results, we are now studying the improved system which will be able to use for detecting fuel leakage from many points. Its result we will be reported in the near future.

\section{References}

[1] J. Darkin and B. Calshaw, Optical Fiber Sensors, vol. 4, ch 17, Artech, Boston, Mass, USA, 1997.

[2] M. Archenault, H. Gagnaire, J. P. Goure, and N. JaffrezicRenault, "A simple intrinsic optical-fibre chemical sensor," Sensors and Actuators B, vol. 8, no. 2, pp. 161-166, 1992.

[3] K. I. Aoyama, K. Nakagawa, and T. Itoh, "Optical time domain reflectometry in a single-mode fiber," IEEE Journal of Quantum Electronics, vol. 17, no. 6, pp. 862-868, 1981.

[4] P. A. Wallace, N. Elliott, M. Uttamlal, A. S. Holmes-Smith, and M. Campbell, "Development of a quasi-distributed optical fibre $\mathrm{pH}$ sensor," in Proceedings of the 14th International Conference on Optical Fiber Sensors (OFS '00), pp. 456-459, October 2000.

[5] A. Weinert, Plastic Optical Fibers, ch 2, Publics MCD, Munich, Germany, 1999.

[6] S. Muto, K. Uchiyama, G. Vishnoi et al., "Plastic optical fiber sensors for detecting leakage of alkane gases and gasoline vapors," in Photopolymer Device Physics, Chemistry, and Applications IV, vol. 3417 of Proceedings of SPIE, pp. 61-69, July 1998.

[7] D. Matsuyama, M. Morisawa, C. X. Liang, and S. Muto, "Plastic Optical Fiber Sensing of Leakage of Fuel in Soil," in Technical Digest of 16th International Conference on Optical Fiber Sensors (OFS-16), Proceedings of SPIE, p. 618, 2003.

[8] M. Morisawa, Y. Amemiya, H. Kohzu, C. X. Liang, and S. Muto, "Plastic optical fibre sensor for detecting vapour phase alcohol," Measurement Science and Technology, vol. 12, no. 7, pp. 877881, 2001. 

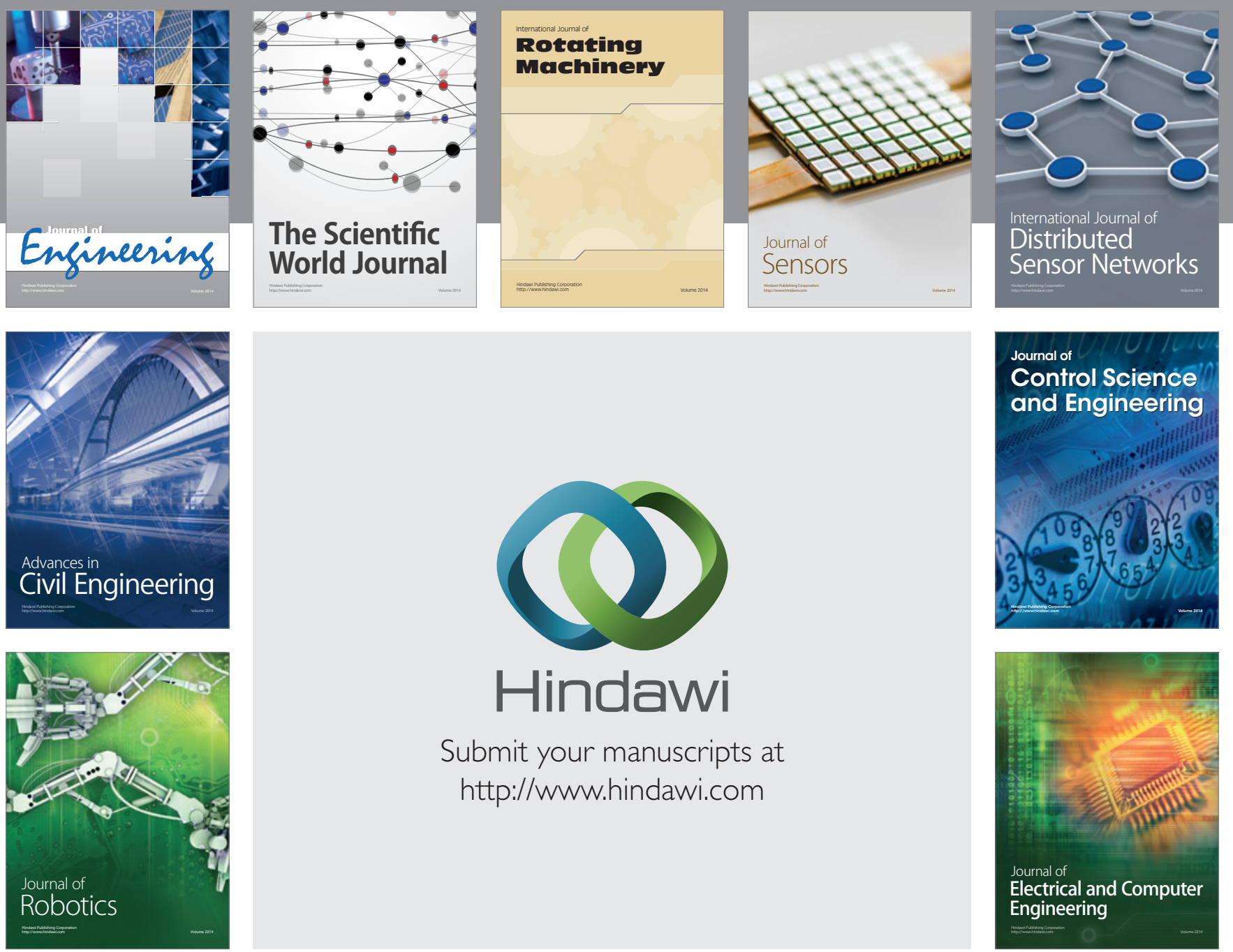

Submit your manuscripts at

http://www.hindawi.com
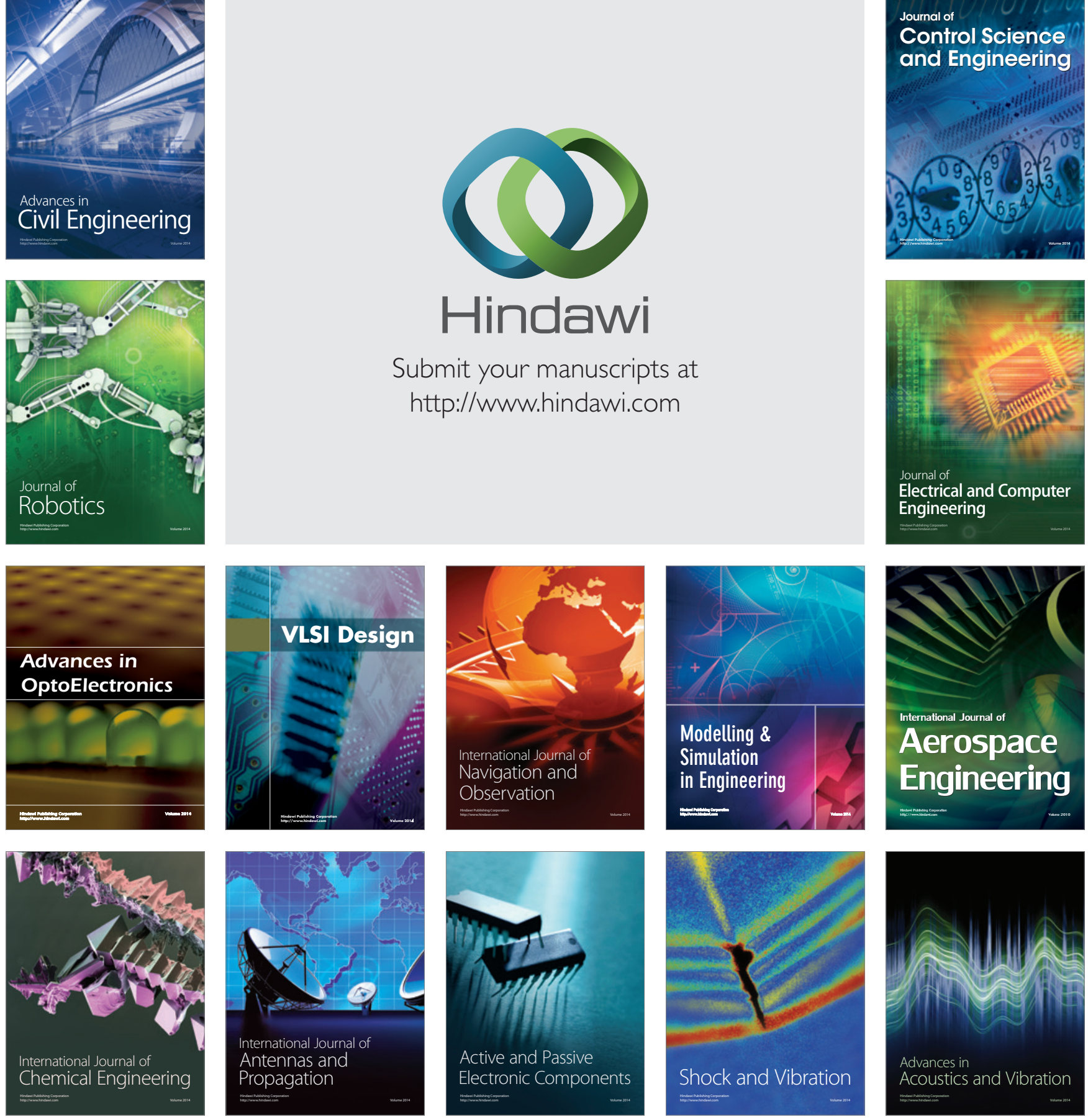